\title{
Elaboración y pertinencia de la matriz de consistencia cualitativa para las investigaciones en ciencias sociales
}

\section{Preparation and Relevance of the Qualitative Consistency Matrix for Research in the Social Sciences}

\section{Mercedes P. Giesecke Sara Lafosse ${ }^{1}$}

Departamento de Antropología, Facultad de Ciencias Sociales, Universidad Nacional Mayor de San Marcos. Lima, Perú

mgieseckes@unmsm.edu.pe ORCID 0000-0001-7603-3202

Citar como: Giesecke, M. (2020). Elaboración y pertinencia de la matriz de consistencia cualitativa para las investigaciones en ciencias sociales. Desde el Sur, 12(2), pp. 397-417.

\section{RESUMEN}

Este trabajo es un aporte para los investigadores y tesistas de ciencias sociales que quieran situar sus proyectos de investigación desde un enfoque cualitativo. La matriz de consistencia cualitativa (MCc) es un instrumento metodológico que posibilita sistematizar, analizar y comprender los procedimientos y avances de una investigación que implica fenómenos, hechos, situaciones y sujetos que difieren en su naturaleza y estructura con los objetos y tópicos trabajados por el enfoque cuantitativo; a diferencia del diseño, estructuración y operativización de variables para validar la pertinencia y viabilidad del proyecto de investigación, que se resume en la verificación y el desarrollo de la matriz de consistencia cuantitativa (MCC) o matriz lógica deductiva.

1 Doctora en Ciencias Sociales con mención en Sociología de la Universidad Nacional Mayor de San Marcos (UNMSM) y magíster en Antropología de la Pontificia Universidad Católica del Perú (PUCP). Licenciada en Antropología (UNMSM), con segunda especialidad en Pedagogía en Filosofía y Ciencias Sociales (PUCP). Exjefa de la Oficina Curricular de la UNMSM (2016), exconsejera de la Facultad de Ciencias Sociales (2016-2018) de esa universidad y coordinadora del Grupo de Investigación «Diseñando el Perú: Estado, ciudadanía, intelectuales y política». Como docente e investigadora, estudia la problemática de la educación intercultural, a través de sus cursos Interculturalidad y Antropología de la Educación, lo que le permite desarrollar proyectos de aprendizaje y servicio solidario en instituciones educativas emblemáticas. Por otro lado, indaga sobre la metodología de la investigación cualitativa, a partir del curso Taller de Metodología para estudiantes de Antropología y las asesorías de tesis de pregrado y posgrado. 


\section{PALABRAS CLAVE}

Investigación cualitativa, diseño de investigación, matriz de consistencia cualitativa

\section{ABSTRACT}

This work is a contribution for researchers and social sciences thesis students who seek to apply a qualitative approach to their research projects. The Qualitative Consistency Matrix (MCC) is proposed as a methodological instrument that makes it possible to systematize, analyze and understand the procedures and advances of research involving phenomena, events, situations and subjects that differ in their nature and structure from those objects and topics addressed via a quantitative approach, in contrast to the design, structuring and operationalization of variables in order to validate the relevance and feasibility of a research project, which is summarized in the verification and development of the Quantitative Consistency Matrix (MCC), or deductive logical matrix.

\section{KEYWORDS}

Qualitative research, research design, qualitative consistency matrix

\section{Introducción}

La presente reflexión parte de mi trayectoria docente en la asesoría de tesis de pregrado y posgrado, así como de los informes de la investigación desarrollados en 2018 por mi equipo de investigación en la Facultad de Ciencias Sociales de la UNMSM.

Los diseños de investigación cualitativos en las ciencias sociales en el Perú están sujetos a los parámetros, las líneas de investigación, las guías metodológicas y los métodos de trabajo que se manejan en cada institución universitaria. Incluso en una misma facultad podemos encontrar diferencias muy marcadas entre los diseños metodológicos que se exigen en cada escuela profesional, y aun en cada asesor que dirige algún proyecto de investigación que conduce a la sustentación de la tesis.

Esta situación produce un cierto desconcierto en el momento en que los tesistas deciden trabajar su investigación desde un enfoque cualitativo, cuyo origen está en el tipo de interacciones entre asesores, ya que por lo general ellos vienen con una disciplina, una trayectoria profesional, un historial académico y una práctica docente que se confrontan con las características equivalentes entre sus pares. Ello se expresa a la hora 
de emitir juicios sobre los avances y procedimientos que se deben seguir con los tesistas, lo que les genera retraso y mayor desorientación, sobre todo de aquellos que vienen de otros campos disciplinares diferentes a las ciencias sociales de carácter cualitativo.

La falta de un consenso sobre la discusión de los criterios básicos para la estructuración de los diseños de investigación con enfoque cualitativo para los tesistas tiene como base el poco diálogo dentro del cuerpo académico, lo que lleva a la reducción individualista que relativiza las asesorías a actos académicos privados, que derivan en la no existencia de matrices de orientación cualitativa en nuestro medio. Con base en la experiencia docente y la registrada y analizada en el estudio de Giesecke (2018a), el presente trabajo tiene por objetivo general la presentación de los criterios que contribuyen a la elaboración de una matriz de consistencia cualitativa $(\mathrm{MCc})$, como alternativa para la organización y sistematización de los niveles de investigación de un mismo fenómeno social (ver Sautu, 2005), la cual apunta a correlacionar las preguntas, las hipótesis y los objetivos de investigación con el método, las técnicas, las fuentes de información y los procedimientos de trabajo a realizar. Finalmente, se complementa con la planificación del trabajo de investigación en sí y el cronograma de entregas, hasta concluir en la sustentación, respetando la naturaleza y las implicancias de cada uno de los niveles dentro del enfoque.

En este trabajo se asume que la propuesta de la construcción de la MCc permite al investigador adecuar a los sujetos de estudio, caracterizados por ser dinámicos y flexibles - algo propio en los estudios de los enfoques cualitativos-, a los cánones de la exigencia y formalidad académicas, por medio de un ordenamiento análogo a los procedimientos que se utilizan en la matriz de consistencia cuantitativa (MCC), con la ventaja de encuadrarla en tres niveles de análisis que se condicionan y retroalimentan: a nivel macro es el histórico o procesual, a nivel meso es el institucional u organizativo, y a nivel micro están las prácticas y percepciones de los sujetos o actores sociales. Es una propuesta de matriz para el uso en el campo de las ciencias sociales, en particular para los estudios de corte antropológico, sociológico, histórico y especialidades afines.

\section{Metodología}

La inspiración para crear y proponer la MCc surgió de la preparación y realización de siete años de clases del Taller de Metodología para la tesis en la Escuela de Antropología de la UNMSM, con un aproximado de 100 tesistas del pregrado, y la praxis como asesora de múltiples tesis de posgrado para el diseño, la implementación y la ejecución de tesis. A la luz de esta experiencia y para validarla se investigó (Giesecke, 2018), por 
medio del método interpretativo y de la investigación, acción que, por su naturaleza flexible y dinámica, permitió registrar las percepciones y los testimonios de los tesistas en torno al uso, las limitaciones y la enseñanza de la metodología cualitativa durante su etapa del pregrado y posgrado en la Facultad de Ciencias Sociales de la UNMSM.

La propuesta tiene como fuente el «Informe final» del «l Ciclo Internacional de Metodología Cualitativa para la Investigación en Ciencias Histórico Sociales: Enfoques, métodos y procedimientos», realizado en la Facultad de Ciencias Sociales de la UNMSM (Giesecke, 2018b), que implicó un proceso de validación de la MCc en las asesorías a 15 tesistas de pregrado y a 10 de posgrado. Otra fuente fue el Informe académico del proyecto de investigación: Métodos cualitativos en estudios históricos sociales (Giesecke, 2018a), aplicado en el posgrado de la Facultad de CCSS, que incluyó 25 entrevistas, 12 aplicadas a los coordinadores y 13 a tesistas de las distintas especialidades que ofrece la facultad.

\section{Resultados}

Durante la sistematización de las entrevistas realizadas a coordinadores y tesistas del posgrado de Ciencias Sociales, se identificaron algunos problemas sumamente importantes directamente relacionados con la elaboración de una investigación cualitativa. Cuestiones en las que se debería basar la formulación de cómo establecer las políticas para ordenar un camino que agilice una comprensión común entre docentes y tesistas para realizar las tesis en tiempos adecuados y con una alta calidad. El diagnóstico arrojó cuatro problemas a tratar en relación con la necesidad de realizar mesas curriculares para discutir sobre metodologías de investigación, hacer la conexión entre la enseñanza metodológica y su uso en las asesorías, responder a la demanda de una mayor interdisciplinariedad, y crear una guía de metodología cualitativa consensuada. En resumen, los principales hallazgos emergen de las voces de los coordinadores de las especialidades de las maestrías y doctorados, como también de los egresados, y son los siguientes:

- Sobre la participación en mesas curriculares para la enseñanza de las metodologías de investigación, en particular de la cualitativa: 9 de 12 docentes coordinadores sí han participado en una de ellas, pero en su mayoría fuera de la universidad, mientras 8 de 13 tesistas no han participado.

- Sobre la guía metodológica para la investigación que se dispone: 9 de 12 coordinadores y 9 de 13 tesistas la señalan como ineficiente, ya que tiene un sesgo exclusivamente cuantitativo, y cuatro tesistas señalan que no la conocen. 
- Sobre si la correspondencia entre las enseñanzas teóricas de las metodologías de la investigación se usan en la realización de las tesis: 7 de 12 coordinadores afirman que no.

- Sobre la demanda por fortalecer la enseñanza de la metodología de la investigación: 9 de cada 12 docentes y 11 de 13 tesistas demandan mayor profundización mediante el mayor soporte metodológico a la investigación.

- Sobre la necesidad de fortalecer el trabajo de investigación interdisciplinario de manera orgánica entre investigadores, docentes y tesistas: 10 de 12 docentes y 12 de 13 tesistas señalan que se debe formular una guía consensuada que se ajuste a la libertad y creatividad de la investigación desde un enfoque cualitativo.

\section{La investigación cualitativa: estado de la cuestión}

En la tradición de las ciencias sociales se señala que la investigación científica tuvo sus raíces en los postulados positivistas de Auguste Comte, según el cual el análisis estadístico de los datos recogidos por medio de estudios y experimentos descriptivos y comparativos serían el camino necesario para llegar a la verdad científica. Por ello, se considera que $E I$ suicidio de Émile Durkheim es tan importante, ya que es uno de los primeros libros que recoge estas pretensiones. Para muchos esta obra francesa sería el primer trabajo sociológico eminentemente científico.

Pero hoy se sabe que ese lugar está reservado para Los obreros europeos (1855) de Frédéric Le Play, quien inicia un verdadero programa de investigación científica al formular e inaugurar el método conocido como estudios de caso. Le Play sustentó sus hallazgos sobre la base de un intenso trabajo de observación participante y comparación sistemática (Ruiz, 2012).

Pero el trabajo de Le Play no es el único antecedente que hace aportes significativos a la investigación científica desde un enfoque cualitativo. Existen textos consistentes que presentan este perfil de investigación, como el de Comunidad y sociedad (1887) de Ferdinand Tönnies, Folkways (1906) de William Graham Sumner, La teoría de la clase ociosa (1899) de Thorstein Veblen, La ética protestante y el espíritu del capitalismo de Max Weber (1905), El hombre marginal (1928) de Robert Park, Los partidos politicos (1911) de Robert Michels y La muchedumbre solitaria (1950) de David Riesman (Ruiz, 2012).

Estos aportes permiten sostener que el valor científico en las investigaciones sociales no solo reside en la cuantificación de los hechos, sino también en la profundidad de la observación y de la descripción, en la documentación y contrastación de las fuentes, en la valoración de los significados de las acciones en sus propios contextos, y en la comparación 
sistemática de los casos a estudiar. Por ello, es importante destacar la producción de las teorías ideográficas en la investigación científica, como paso previo a las teorías nomotéticas. Cuantos más casos sean explicados por una teoría, más nomotético se convertirá el análisis (Bernard, 1995).

El investigador que desee emprender un proyecto de investigación cualitativo debe saber que el conocimiento adquiere un carácter constructivo e interpretativo. Esto es afirmar que el conocimiento es una producción humana y no es algo que ya está listo para conocerse, como si fuera una realidad previamente ordenada de acuerdo con categorías universales. De ahí que en el proceso de investigación se generen nuevas zonas de sentido, esto visto como espacios de inteligibilidad que se producen en el proceso de la investigación científico social, que abren la posibilidad de la profundización en el tema abordado, y, por lo tanto, en su conjunto generan un espacio nuevo de creación de mayor conocimiento.

La investigación cualitativa es dinámica, porque la hipótesis del investigador está asociada a un modelo teórico que mantiene una constante tensión con el momento empírico y cuya legitimidad radica en la capacidad del modelo para ampliar y profundizar su conocimiento (González Rey, 2006, pp. 24-31). Esta profundización como proceso tendría cinco niveles: se inicia desde que el investigador recoge los datos, luego se plantea las preguntas o modifica las previas, empieza a formar categorías, busca patrones para fundamentar la teoría y, finalmente, construye la teoría con base en el caso (Coutinho, 2011). Estos puntos permiten sostener la necesidad de desarrollar distintas formas de abordar los fenómenos sociales desde lo que Martin Packer (2013) denomina la ciencia de la investigación cualitativa $(\mathrm{CIC})$.

Esta CIC toma a los seres humanos no como objetos, sino como seres culturales e históricos específicos y que hacen de sí tipos particulares de objetos cualitativos. La CIC sería capaz de captar esta constitución humana a la luz de un realismo plural en contextos y procesos concretos. Desde esta propuesta, no se trata de discutir sobre cuán objetiva o neutral es la investigación, ya que ello hace ver a los actores involucrados como objetos indiferentes ante el investigador. Por el contrario, se trata de reconocer que las investigaciones o estudios de caso también deben ser evaluados desde la dimensión ética de quien los lleva a cabo (Packer, 2013, p. 6).

La CIC fijará su mirada crítica en la manipulación y eficacia del método, cayendo en un instrumentalismo, y también, en palabras de Coutinho (2011), cuestionará los fundamentos de los métodos, las filosofías y las teorías que subyacen e influyen sobre las elecciones que toma el investigador. La metodología cualitativa analiza y describe los métodos, y se aleja de la práctica para poder construir teorías de importancia científico social. 
A nivel conceptual, el objeto de estudio en la investigación de corte cualitativo no son los comportamientos, sino las intenciones y situaciones; es decir, se trata de investigar las ideas, de descubrir significados en las acciones individuales y en las interacciones sociales a partir de la perspectiva de los actores que intervienen en el proceso (Coutinho, 2011, p. 12).

A nivel metodológico, la investigación de índole cualitativa se basa en el método inductivo, ya que la finalidad última es comprender y evidenciar las intenciones, las motivaciones y los propósitos en el mismo contexto de producción de significados. Es recoger los datos desde el mapa significativo de los actores.

\section{El diseño de investigación}

Implica modelar, planificar y estructurar los procesos y procedimientos que se van a seguir para llevar a cabo una investigación. Es decidir qué enfoques, métodos e instrumentos son los más pertinentes según el objeto o sujeto de investigación. En ese sentido, se requiere describir todas las acciones que se van a realizar. Sin embargo, hay que tomar en cuenta que el diseño planificado como tal es una hoja de ruta o marco orientador en la investigación. En la investigación cualitativa, el diseño metodológico tiene un carácter flexible, mas nunca prescriptivo, debido a la naturaleza social de los tópicos que aborda.

También se puede considerar como una declaración acerca del tipo de investigación que se realiza; una afirmación de la unidad de estudio y de los actores o sujetos estudiados, con su correspondiente agrupación en estamentos - si correspondiera-; una definición de los instrumentos utilizados para recoger la información y definición conceptual y operacional de las categorías y subcategorías apriorísticas; por último, una orientación de los procedimientos centrales que guían el proceso de análisis de la información.

La importancia del diseño de investigación se centra en la necesidad de organizar constantemente las ideas, lo que, en palabras de Bourdieu (2002), sería la jerarquización de los actos epistemológicos. Esta estrategia contribuye a construir el objeto de estudio, lo que de manera esquemática plantea Bernard (1995) en cuatro pasos: primero, se formula un problema teórico; a continuación, se seleccionan el sitio y el método apropiados; luego, se recolectan datos y se los analiza; $y$, finalmente, se rebate o confirma la proposición teórica que impulsó la investigación.

En esa línea, Murcia (2003) propone dos momentos en la investigación: en el primero se da la reflexión desde la realidad preconfigurada, a partir de la cual surge la perspectiva inductiva y el tema de investigación; $y$, en un segundo momento, se desarrolla el diseño metodológico para la 
investigación: la manera en que se va a estructurar la guía para investigar, considerando las interrogantes, el sentido general de la investigación, los objetivos y métodos para el recojo de información y el trabajo de campo.

Por ello, la investigación cualitativa no está determinada exclusivamente por los postulados teóricos que pretenden generalizar las observaciones específicas, de las que se quiere dar cuenta, sino que ella misma, conjuntamente con el proceso de campo, va construyendo su propia interpretación de la realidad investigada. La teoría aquí es un resultado de la propia investigación cualitativa, que puede afirmar, negar, descubrir o comparar la data y su significado. Esta forma de concebir y comparar el rol de la teoría en las investigaciones cualitativas y cuantitativas es seguida por Sierra, quien sostiene que «la investigación cualitativa, por medio de un diseño del proceso indagativo, abierto y flexible, ofrece una imagen holográmica de la sociedad en movimiento» (Sierra, 1998, p. 303).

Recapitulando, el diseño de la investigación cualitativa debe tener un carácter adaptable a las exigencias de la realidad empírica, que es de donde se extraen los datos para postular e inferir inductivamente cierto cuerpo de ideas que concluyan en la generación de teorías. Las explicaciones cualitativas son generalizaciones de sujetos en un tiempo, en un lugar y con un lenguaje determinado, donde las teorías preestablecidas o preelaboradas son un soporte que proveen conceptos formativos, ordenadores y significados de comprensión que son útiles para realizar el camino inicial de toda investigación, debido a la imposibilidad de partir de cero. En el trayecto de profundización de la investigación, todo el acervo teórico estará sujeto a revisión, reformulación o restructuración, es decir, estará supeditado a una vigilancia epistemológica progresiva (Bourdieu, 2002), hasta lograr una adecuada interpretación teórica de aquello que se está investigando.

En ese sentido, es mejor hablar de un «contexto conceptual» antes de un marco teórico en sí, como sugiere Mendizábal (2012, citado en Izcara, 2014, p. 25). Para él estamos ante un marco teórico cuando se sigue un modelo hipotético-deductivo, de teorías validadas, que buscan confirmar o negar el objeto estudiado. En cambio, se menciona que se opera o se parte de un «contexto conceptual», cuando las conclusiones o formulaciones sostenidas en teorías previas se delimitan para explicar los datos e información recogidos durante la investigación de campo.

Entre los diseños de investigación cualitativa más usados están la teoría fundamentada, la etnografía, la exploración narrativa, la investigaciónacción y el enfoque fenomenológico. Si bien teóricamente guardan algunas diferencias en cuanto a sus planteamientos y formas de proceder, se observa que dichos diseños comparten las técnicas y los instrumentos 
para el recojo de datos empíricos, ya que, en última instancia, se tiene un interés por los significados, las acciones y los lenguajes que pueda producir, en diferentes situaciones o contextos, el ser humano. La investigación cualitativa es humanista, en el sentido de que intenta describir, explorar, comparar, comprender e intuir desde la mirada de los actores sociales. A continuación, se observa los principales matices entre los diseños mencionados:

TABLA 1. Diseño de investigación

\begin{tabular}{|c|c|c|}
\hline \multicolumn{3}{|c|}{ Diseños de investigación en investigación cualitativa } \\
\hline $\begin{array}{l}\text { Tipo de } \\
\text { diseño }\end{array}$ & Fundamentos & Alcances \\
\hline 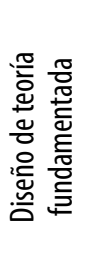 & $\begin{array}{l}\text { Se basa en el interaccionismo simbóli- } \\
\text { co. Su planteamiento básico es que las } \\
\text { proposiciones teóricas surgen de los } \\
\text { datos obtenidos en la investigación, } \\
\text { más que de los estudios previos. Es } \\
\text { el procedimiento el que genera el en- } \\
\text { tendimiento de un fenómeno social. }\end{array}$ & $\begin{array}{l}\text { Útil cuando las teorías disponibles no explican } \\
\text { el fenómeno o planteamiento del problema, } \\
\text { o bien cuando no cubren a los participantes } 0 \\
\text { muestra de interés (Creswell, 2005). }\end{array}$ \\
\hline 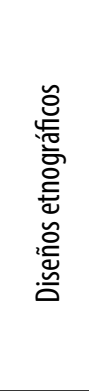 & $\begin{array}{l}\text { Buscan describir y analizar ideas, } \\
\text { creencias, significados, conocimientos } \\
\text { y prácticas de grupos, culturas y co- } \\
\text { munidades. Incluso pueden ser muy } \\
\text { amplios y abarcar la historia, la geo- } \\
\text { grafía y los subsistemas socioeconó- } \\
\text { mico, educativo, político y cultural de } \\
\text { un sistema social (rituales, símbolos, } \\
\text { funciones sociales, parentesco, migra- } \\
\text { ciones, redes, entre otros). }\end{array}$ & $\begin{array}{l}\text { Su alcance está sujeto al tipo o la dimensión } \\
\text { de estudio que se quiere realizar. Aquí encon- } \\
\text { tramos: etnografías procesales, etnografía } \\
\text { holística o clásica, etnografía particularista, } \\
\text { etnografía de corte transversal y etnografía } \\
\text { etnohistórica. }\end{array}$ \\
\hline 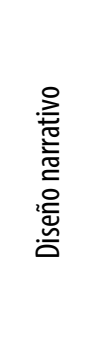 & $\begin{array}{l}\text { El investigador recolecta datos sobre } \\
\text { las historias de vida y experiencias de } \\
\text { determinadas personas para descri- } \\
\text { birlas y analizarlas, teniendo en cuen- } \\
\text { ta el contexto social y físico donde se } \\
\text { desarrollan. }\end{array}$ & $\begin{array}{l}\text { Los datos se obtienen de autobiografías, bio- } \\
\text { grafías, entrevistas, documentos, artefactos y } \\
\text { materiales personales y testimonios (que en } \\
\text { ocasiones se encuentran en cartas, diarios, } \\
\text { artículos en la prensa, grabaciones radiofóni- } \\
\text { cas y televisivas, entre otros). Pueden referirse } \\
\text { a toda la historia de la vida de una persona } 0 \\
\text { grupo, un pasaje o época de dicha historia de } \\
\text { vida, } 0 \text { uno o varios episodios. }\end{array}$ \\
\hline
\end{tabular}


Resolver problemas cotidianos e in- Los participantes que están viviendo un promediatos, y mejorar prácticas concreblema son los que están mejor capacitados tas. Su propósito fundamental se cen- para abordarlo en un entorno naturalista. La tra en aportar información que guíe la conducta de estas personas está influida de toma de decisiones para programas, manera importante por el entorno natural en procesos y reformas estructurales. que se encuentran. La metodología cualitativa es la mejor para el estudio de los entornos naturalistas.

\begin{tabular}{|c|c|c|}
\hline 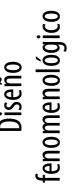 & $\begin{array}{l}\text { Se enfocan en las experiencias indivi- } \\
\text { duales subjetivas de los participantes. }\end{array}$ & $\begin{array}{l}\text { Responden a la pregunta: ¿cuál es el significa- } \\
\text { do, la estructura y la esencia de una experien- } \\
\text { cia vivida por una persona (individual), grupo } \\
\text { (grupal) o comunidad (colectiva) respecto de } \\
\text { un fenómeno? }\end{array}$ \\
\hline
\end{tabular}

Fuente: Salgado (2007). Investigación cualitativa. Diseños, evaluación del rigor metodológico y retos.

Elaboración: Mercedes Giesecke S.

TABLA 2. Procedimientos para el diseño de investigación

\begin{tabular}{ll} 
Aspectos & $\begin{array}{l}\text { Contenidos } \\
\text { El problema de investigación }\end{array}$ \\
$\begin{array}{ll}\text { Se presenta y justifica el problema de investigación } \\
\text { expresado en una interrogante. }\end{array}$ \\
\hline El método de investigación & Se fundamenta el método de investigación utilizado. \\
\hline Los objetivos & $\begin{array}{l}\text { Se presentan los objetivos generales y específicos de } \\
\text { la investigación. }\end{array}$ \\
\hline Las hipótesis (si se considera necesario) & Se enumeran las hipótesis. \\
\hline Las variables o categorías de estudio & Se enumeran las variables de estudio. \\
\hline La muestra de estudio & Se especifican el contexto y la muestra de estudio. \\
\hline Las técnicas e instrumentos para recoger & $\begin{array}{l}\text { Se presentan, explican y justifican las técnicas y los } \\
\text { instrumentos utilizados. }\end{array}$ \\
la información & $\begin{array}{l}\text { Se explica el proceso seguido para organizar, clasificar, } \\
\text { analizar e interpretar los datos recogidos. }\end{array}$ \\
\hline Las técnicas e instrumentos para analizar \\
la información
\end{tabular}

Fuente: Díaz, Suárez y Flores (2016). Guía de investigación en educación.

Elaboración: Mercedes Giesecke $S$. 


\section{Discusión: propuesta para la construcción de la MCc}

El instrumento conocido como matriz de consistencia nace como una herramienta metodológica para ordenar, jerarquizar, estructurar y controlar los conceptos, las categorías, las dimensiones y las variables, entre el objeto o fenómeno que se quiere estudiar y los atributos que se le asignan. Es decir, parte del principio de no contradicción y de identidad, como lo establece la lógica formal. Es un cuadro de doble entrada compuesto por columnas y filas, que posibilita al investigador analizar y evaluar el grado de coherencia y conexión lógica entre el problema planteado, los objetivos propuestos, las posibles repuestas, los atributos (variables) que se quiere analizar y todos los elementos que se utilizan en el diseño y el método de investigación (Marroquín, 2012 y Carrasco, 2018).

Esta forma de presentar la matriz de consistencia también es conocida como MCC y es el modelo que más se usa en los proyectos de investigación de las distintas especialidades. Al tener varias características de la exigencia de la lógica causal y partir del modelo hipotético deductivo de Karl Popper, se presume que los fenómenos a estudiar, en este caso de las ciencias sociales, podrían responder y explicarse partiendo de las investigaciones previamente realizadas, por lo cual las teorías marcan el derrotero de la confirmación o negación de los presupuestos planteados.

Para construir la MCC, lo primero que se realiza es identificar el tipo de variable con que se está trabajando, la cual se divide por el tipo de relación dependiente, independiente e interdependiente y por su naturaleza cualitativa y cuantitativa. La variable se define por los atributos, las características o las propiedades que puede presentar una persona o cosa, que varían en cada una de ellas en diferentes momentos o circunstancias. Además, estas deben ser susceptibles de medición (por ejemplo, talla de jóvenes, presión arterial, sueldo mínimo, productividad laboral, coeficiente intelectual, etc.).

Una vez que se escogió con qué variable se cuenta o se va trabajar, se empieza a evaluar su consistencia, coherencia y conexión lógica en un cuadro conocido como matriz de operacionalización de la variable (MOV), y es donde se describe el tipo de variable, su definición conceptual, las dimensiones que se van a analizar, los indicadores a medir y los instrumentos con los que se validará la medición, como vemos en el ejemplo presentado a continuación: 
TABLA 3. Ejemplo de MOV

\begin{tabular}{|c|c|c|c|c|c|}
\hline Variables & $\begin{array}{l}\text { Tipos de } \\
\text { variable }\end{array}$ & $\begin{array}{l}\text { Definición } \\
\text { conceptual }\end{array}$ & Dimensiones & Indicadores & Instrumentos \\
\hline \multirow{5}{*}{ 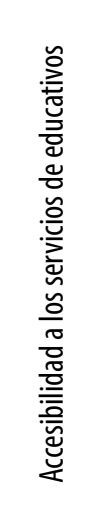 } & \multirow{5}{*}{ 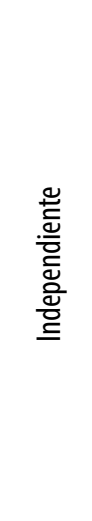 } & \multirow{5}{*}{$\begin{array}{l}\text { Mayor } 0 \\
\text { menor } \\
\text { posibilidad } \\
\text { de tomar } \\
\text { contacto } \\
\text { con los } \\
\text { servicios } \\
\text { educativos } \\
\text { para } \\
\text { recibir } \\
\text { asistencia }\end{array}$} & $\begin{array}{l}\text { Accesibilidad } \\
\text { geográfica }\end{array}$ & $\begin{array}{l}\text { Tiempo medido en horas y } \\
\text { minutos que tarda una per- } \\
\text { sona en trasladarse desde } \\
\text { su domicilio a la institución } \\
\text { educativa }\end{array}$ & \multirow{5}{*}{$\begin{array}{l}\text { Cuestionario } \\
\text { Encuestas } \\
\text { Entrevistas }\end{array}$} \\
\hline & & & $\begin{array}{l}\text { Accesibilidad } \\
\text { económica }\end{array}$ & $\begin{array}{l}\text { Cantidad de dinero que gasta } \\
\text { para recibir atención }\end{array}$ & \\
\hline & & & \multirow{3}{*}{$\begin{array}{l}\text { Accesibilidad } \\
\text { cultural }\end{array}$} & Disponibilidad económica & \\
\hline & & & & $\begin{array}{l}\text { Conocimientos sobre el servi- } \\
\text { cio educativo }\end{array}$ & \\
\hline & & & & $\begin{array}{l}\text { Percepción sobre el problema } \\
\text { del servicio educativo }\end{array}$ & \\
\hline
\end{tabular}

Fuente: Elaboración propia

Una vez realizada la operacionalización de la variable y definidas las dimensiones que se quieren indagar, se pasa a construir la MCC, que, a diferencia de la MOV, incluye los problemas, los objetivos, la hipótesis, la metodología y la población sujeta a la investigación. Lo que se busca aquí es que tanto las características generales como las específicas tengan relación de concordancia y trazabilidad metodológica entre el problema, el objetivo y la hipótesis (la hipótesis sí es un elemento determinante desde el diseño hasta la verificación o su negación). No se concibe la MCC sin establecimiento previo de la hipótesis. En el siguiente cuadro se observa un ejemplo del uso de la MCC con dos variables dependientes: 
TABLA 4. MCC

\begin{tabular}{|c|c|c|c|c|c|}
\hline Problemas & Objetivos & Hipótesis & Variable & Metodología & Población \\
\hline $\begin{array}{l}\text { Problema } \\
\text { general }\end{array}$ & Objetivo general & Hipótesis general & $\begin{array}{l}\text { Variable } \\
\text { I }\end{array}$ & Tipo & \multirow{6}{*}{$\begin{array}{l}\text { La po- } \\
\text { blación: } \\
\text { estu- } \\
\text { diantes } \\
\text { de la I. E. } \\
\text { Mariano } \\
\text { Melgar } \\
\text { La } \\
\text { muestra: } \\
\text { alumnos } \\
\text { del } \\
\text { primer, } \\
\text { segundo } \\
\text { y tercer } \\
\text { grado de } \\
\text { secun- } \\
\text { daria de } \\
\text { la I. E. } \\
\text { Mariano } \\
\text { Melgar }\end{array}$} \\
\hline $\begin{array}{l}\text { ¿En qué medi- } \\
\text { da el contexto } \\
\text { de enseñanza } \\
\text { con materiales } \\
\text { lúdicos en cien- } \\
\text { cias sociales se } \\
\text { relaciona con el } \\
\text { aprendizaje de } \\
\text { los alumnos de } \\
\text { la I. E. Mariano } \\
\text { Melgar? }\end{array}$ & $\begin{array}{l}\text { Establecer la re- } \\
\text { lación que existe } \\
\text { entre el contexto } \\
\text { de enseñanza } \\
\text { con materiales } \\
\text { lúdicos en cien- } \\
\text { cias sociales con } \\
\text { el aprendizaje de } \\
\text { los alumnos de } \\
\text { la I. E. Mariano } \\
\text { Melgar. }\end{array}$ & $\begin{array}{l}\text { El contexto de } \\
\text { enseñanza con } \\
\text { materiales lúdi- } \\
\text { cos en ciencias } \\
\text { sociales se rela- } \\
\text { ciona significati- } \\
\text { vamente con el } \\
\text { aprendizaje de } \\
\text { los alumnos de } \\
\text { la I. E. Mariano } \\
\text { Melgar. }\end{array}$ & $\begin{array}{l}\text { El con- } \\
\text { texto de } \\
\text { la ense- } \\
\tilde{n} \text { a } \mathrm{n} \mathrm{z} \mathrm{a} \\
\text { con ma- } \\
\text { teriales } \\
\text { lúdicos. }\end{array}$ & \multirow{5}{*}{$\begin{array}{l}\text { Nivel de in- } \\
\text { vestigación: } \\
\text { básico- } \\
\text { aplicativo } \\
\text { Tipo de in- } \\
\text { vestigación: } \\
\text { descriptivo- } \\
\text { correlacio- } \\
\text { nal, de corte } \\
\text { transversal } \\
\text { Diseño de la } \\
\text { inves- } \\
\text { tigación: } \\
\text { descriptivo- } \\
\text { correlacional }\end{array}$} & \\
\hline $\begin{array}{l}\text { Problema } \\
\text { específico (1) }\end{array}$ & $\begin{array}{l}\text { Objetivo } \\
\text { específico (1) }\end{array}$ & $\begin{array}{l}\text { Hipótesis } \\
\text { específica (1) }\end{array}$ & $\begin{array}{l}\text { Variable } \\
\text { II }\end{array}$ & & \\
\hline $\begin{array}{l}\text { ¿De qué mane- } \\
\text { ra el contexto } \\
\text { de enseñanza } \\
\text { con materiales } \\
\text { lúdicos en cien- } \\
\text { cias sociales se } \\
\text { relaciona con el } \\
\text { aprendizaje de } \\
\text { los alumnos de } \\
\text { la I. E. Mariano } \\
\text { Melgar? }\end{array}$ & $\begin{array}{l}\text { Establecer la } \\
\text { relación del con- } \\
\text { texto de ense- } \\
\text { ñanza con mate- } \\
\text { riales lúdicos en } \\
\text { ciencias sociales } \\
\text { con el aprendiza- } \\
\text { je de los alumnos } \\
\text { de la I. E. Maria- } \\
\text { no Melgar. }\end{array}$ & $\begin{array}{l}\text { El contexto de } \\
\text { enseñanza con } \\
\text { materiales lúdi- } \\
\text { cos en ciencias } \\
\text { sociales se rela- } \\
\text { ciona significati- } \\
\text { vamente con el } \\
\text { aprendizaje de } \\
\text { los alumnos de } \\
\text { la I. E. Mariano } \\
\text { Melgar. }\end{array}$ & $\begin{array}{l}\text { Aprendi- } \\
\text { zaje }\end{array}$ & & \\
\hline $\begin{array}{l}\text { Problema } \\
\text { específico (2) }\end{array}$ & $\begin{array}{l}\text { Objetivo } \\
\text { específico (2) }\end{array}$ & $\begin{array}{l}\text { Hipótesis } \\
\text { específica (2) }\end{array}$ & & & \\
\hline $\begin{array}{l}\text { Problema } \\
\text { específico (3) }\end{array}$ & $\begin{array}{l}\text { Objetivo } \\
\text { específico (3) }\end{array}$ & $\begin{array}{l}\text { Hipótesis } \\
\text { específica (3) }\end{array}$ & & & \\
\hline
\end{tabular}

Fuente: Elaboración propia

Así como en la investigación cuantitativa se hace uso de una MCC, la misma idea se adapta a las necesidades de la investigación cualitativa, pero con ciertas características que permiten adecuarla a fenómenos que se tornan complicados de dimensionar y cuantificar, como la experiencia, las percepciones o las sensaciones sobre algún hecho, circunstancias, acciones o acontecimientos de los cuales se formó o se forma parte. La producción del conocimiento que emerge de la investigación cualitativa tiene un carácter siempre dialógico, porque también está mediada por las significaciones que posee el investigador sobre lo que está investigando. 
Como lo estipulan las consideraciones de González Rey (2007) sobre la investigación cualitativa, al señalar que la calidad de una investigación depende del compromiso del autor con lo que está investigando, lo que cuenta es el papel activo del investigador para estimular a los participantes y persuadirlos de que expresen su subjetividad, así como motivar su curiosidad e inquietud, de tal manera que deseen colaborar con lo que se interesa conocer.

La MCc comparte ciertos elementos de la estructura de la MCC, pero el concepto de la matriz cualitativa incluye otro conjunto de aspectos, con distinciones, formas, procedimientos y metodologías cualitativas que se presentan a continuación:

a) Articulación de los niveles de investigación dentro de un mismo sujeto de estudio: la MCc toma en consideración hasta tres marcos de referencia contextual y situacional donde está inscrito el sujeto de estudio: el nivel macrosocial, el nivel mesosocial y el nivel microsocial. Esta estrategia permite observar, a modo de mapa panorámico, dónde incidir con mayor rigurosidad, así como identificar en dónde se ubica el contexto inmediato y en qué aspectos se debe fijar el foco de atención en el estudio de caso. Por ejemplo, si mi investigación es «El proceso de formación de la doctrina militar», debo considerar que el nivel microsocial son las prácticas de instrucción, los rituales castrenses y la educación técnico-académico dentro de las escuelas militares. El nivel meso o intermedio lo constituirá la institución militar en su conjunto, como puede ser el Ejército peruano, donde están las otras áreas que influyen a la formación castrense, pero que no tiene incidencia determinante, mas sí condicionante. Finalmente, tenemos el nivel macrosocial, que está representado por el sistema conjunto de seguridad nacional, que ordena los lineamientos generales de la política de defensa, así como por su contexto sociohistórico. 
TABLA 5. Niveles de investigación en la MCc

Nivel macrosocial

Toma en cuenta que los sujetos están enmarcados en estructuras mayores y obedecen a macropatrones que influyen en sus acciones. Se hace necesario entender cómo está configurado el sistema social, lo cual obedece analíticamente a lo que llamamos nivel macrosocial.

\begin{tabular}{|c|c|c|c|c|}
\hline $\begin{array}{l}\text { Nivel mesosocial } \\
\text { Se refiere a un nivel intermedio, no tan } \\
\text { inmediato al sujeto, pero que le es más } \\
\text { próximo que el nivel macrosocial. Este } \\
\text { es su entorno, del cual recibe un influ- } \\
\text { jo más directo. Llamamos a este nivel } \\
\text { mesosocial. }\end{array}$ & $\begin{array}{l}\text { La pregunta } \\
\text { intermedia }\end{array}$ & $\begin{array}{l}\text { La hipótesis } \\
\text { intermedia }\end{array}$ & $\begin{array}{l}\text { El objetivo } \\
\text { interme- } \\
\text { dio }\end{array}$ & $\begin{array}{l}\text { Correspon- } \\
\text { dientes } \\
\text { métodos y } \\
\text { técnicas de } \\
\text { investiga- } \\
\text { ción }\end{array}$ \\
\hline $\begin{array}{l}\text { Nivel microsocial } \\
\text { Es el nivel individual en donde se } \\
\text { plantea el estudio y del cual se quiere } \\
\text { conocer sus interrelaciones tanto entre } \\
\text { los actores, sus redes y con los otros ni- } \\
\text { veles macro y meso, pues entendemos } \\
\text { que el actor influye en su medio como } \\
\text { su medio en él. }\end{array}$ & $\begin{array}{l}\text { La pregunta } \\
\text { específica }\end{array}$ & $\begin{array}{l}\text { Hipótesis } \\
\text { específica }\end{array}$ & $\begin{array}{l}\text { Objetivo } \\
\text { específico }\end{array}$ & $\begin{array}{l}\text { Correspon- } \\
\text { dientes } \\
\text { métodos y } \\
\text { técnicas de } \\
\text { investiga- } \\
\text { ción propia- } \\
\text { mente de } \\
\text { campo }\end{array}$ \\
\hline
\end{tabular}

b) Las preguntas de investigación en la investigación cualitativa (IC): en primer término, se presenta la pregunta de investigación, que expone sintéticamente una problemática, que será interpelada para conocerla. Las preguntas deben ser hechas de manera concisa y no ser generales. Deben ayudar a clarificar e intentar vincular los macroprocesos con las experiencias del sujeto. Corresponden a los tres niveles socioestructurales a investigar.

c) Las hipótesis en la IC: adquieren un carácter flexible, general, emergente y contextual, es decir, se fortalecen según se vayan sistematizando y analizando los datos recogidos en el trabajo de campo, ya que las hipótesis en sí mismas son un producto de la investigación (Hernández, Fernández y Batista, 2010). Ellas adquieren la forma de una hipótesis de trabajo y, por lo general, no son obligatorias en investigaciones de corte descriptivo-exploratorio. Sin embargo, en las investigaciones comparativas las hipótesis sí juegan un rol importante para demarcar los criterios y los rasgos que se quieren conocer, ya que permiten inferir a priori que lo que se quiere comparar tiene ciertas características que las hace plausibles de ser analizadas. Aquí no se parte de cero; de antemano, el investigador hizo una discriminación 
y selección de lo que se quiere comparar, mediante investigaciones previas o un trabajo de precampo.

En esa línea de pensamiento se encuentra lo afirmado por Sautu (2005), quien establece que toda investigación que busca dar respuesta a los casos particulares, a la experiencia y acciones de los actores y a las percepciones que se producen en plano de la vida cotidiana, necesariamente debe partir de un razonamiento inductivo. En ese sentido, la hipótesis en la IC refuerza su consistencia en la medida en que los datos de los casos particulares muestren nuevas evidencias posibles de ser generalizadas.

d) El objetivo en la IC: en principio debe expresar de manera clara y concisa lo que se quiere lograr con la investigación. Estos objetivos deben tener relación con el enfoque o diseño de investigación empleado. Si uno realiza una IC, por lo general se situará en un análisis descriptivo, exploratorio, analítico o comparativo; en tal sentido, los verbos infinitivos a utilizar en relación con sus objetivos de la acción indagatoria deben estar acordes a los alcances que se quieren lograr. Por otra parte, se advierte que se deben evitar los verbos que aludan a acciones finales o explicativos, ya que no se puede garantizar las consecuencias futuras.

TABLA 6. Tipos de verbos en los objetivos de la investigación cualitativa (IC)

\begin{tabular}{lllllll} 
Descriptiva & Exploratoria & \multicolumn{1}{c}{ Analítica } & Comparativa & $\begin{array}{c}\text { Se debe evitar } \\
\text { el uso de verbos } \\
\text { explicativos }\end{array}$ & $\begin{array}{l}\text { Se debe evitar el } \\
\text { uso de verbos de } \\
\text { acciones finales }\end{array}$ \\
\hline Calcular & Conocer & Criticar & Contrastar & Comprobar & Capacitar \\
\hline Analizar & Definir & Descomponer & Diferenciar & Demostrar & Enseñar \\
\hline Caracterizar & Descubrir & Analizar & Comparar & Determinar & Cambiar \\
\hline Clasificar & Detectar & Establecer & Asociar & Establecer & Mejorar \\
\hline Cuantificar & Estudiar & & Asemejar & Evaluar & Motivar \\
\hline Describir & Explorar & & & Explicar & \\
\hline Examinar & Indagar & & & Verificar & \\
\hline Identificar & Sondear & & & & \\
\hline
\end{tabular}

Fuente: Elaboración propia

El objetivo es el que marca los pasos procedimentales de la investigación cualitativa, que viene determinado por la pregunta e hipótesis de investigación, como un apoyo que da una pauta metodológica, en los pasos y acciones a seguir y para indagar los puntos claves que ayuden a corroborar o anular la hipótesis. Las actividades que nacen del objetivo deben ser rigurosas en la aplicación del método científico y con un conocimiento ético de la investigación. 
e) La metodología en la IC: en la MCc se ha considerado la columna que describe la metodología a seguir, que comprende el enfoque cualitativo de la investigación, las técnicas y herramientas, como las entrevistas no dirigidas, la observación participante, los grupos focales, la revisión documental, etc.; incluye instrumentos como los cuestionarios, las fichas de observación y bibliográficas y otros, con los cuales se recabarán los datos empíricos que son los insumos de toda investigación.

En relación con la técnica, Tamayo (2003) sostiene que está compuesta por un conjunto de mecanismos, sistemas o medios que ayudan a investigar, dirigir, conservar, rediseñar y transmitir los datos recogidos, ya sea de gabinete o del trabajo de campo. Contiene, además, las normas o los lineamientos que auxilian al método. Dichas técnicas son valoradas por su funcionalidad al garantizar la mejora de los procesos, el ahorro de tiempo y el manejo de los recursos.

En la MCc se han mantenido la pregunta, la hipótesis y los objetivos, los cuales son las piezas fundamentales de toda investigación científica. Se ha eliminado la parte de variables en tanto la realidad social no sigue una causalidad lineal sino recursiva, en donde varios elementos permanentemente influyen sobre otros y presentan retroalimentación y ajustes particulares, con lo cual el modelo de proponer variables se hace limitado y engañoso.

La MCc se caracteriza por ser un organizador sistémico de los diversos elementos que intervienen en la investigación cualitativa. Es un instrumento que ayuda a tener una «vigilancia epistemológica» sobre el objeto o sujeto de estudio cualitativo.

El modelo que presento a continuación ha sido muy útil en todos estos años para acelerar los procesos de elaboración de tesis, al contribuir a una mejor acotación del tema a investigar y a organizar la profusa data recogida por tesistas, e incluso a identificar los vacíos que restan por investigar.

Presento el modelo de MCc, que se explica mediante la leyenda descriptiva: 
TABLA 7. Matriz de consistencia cualitativa

MATRIZ DE CONSISTENCIA CUALITATIVA

I $\quad$ III

NIVEL SOCIOES- 1. PLANTEAMIENTO DEL PROBLEMA (y TRUCTURAL justificación)

2. PREGUNTAS

3. HIPÓTESIS (en caso necesario)

4. OBJETIVOS DE INVESTIGACIÓN (0 tareas que se desprenden de las hipótesis)

5. ÍNDICE

Se prepara el índice respetando los niveles socioestructurales y a partir de los objetivos. El índice integra todos los niveles.

Preguntas, hipótesis, objetivos para

cada nivel socioestructural:

GENERAL

INTERMEDIA

ESPECÍFICA
SE ORGANIZA LA DATA SEGÚN OBJETIVOS DE INVESTIGACIÓN Y NIVELES SOCIOESTRUCTURALES:

6. BIBLIOGRAFÍA

\section{MÉTODOS Y TÉCNICAS:}

Antecedentes y contexto consideran aspectos epistemológicos, teóricos, históricos, geográficos, metodológicos, a partir de entrevistas, historias de vida, cuestionarios, grupos focales, sistematizaciones, observación participante; avanza con la redacción, etc.

8. CRONOGRAMA DE ACTIVIDADES SEGÚN OBJETIVO 9. AVANCES Y FECHAS DE ENTREGA

Leyenda: MATRIZ DE CONSISTENCIA CUALITATIVA (MCc)

Las columnas I y II indican la secuencia lógica que debe recorrer el proceso de investigación, que inicia en el análisis del nivel de estudio más abstracto y general. El tipo de pregunta, hipótesis y objetivos planteados de manera lógica y coherente corresponden para los tres niveles socioestructurales, lo cual debe concordar con los métodos, las técnicas, la programación de actividades, la selección de referencias bibliográficas y la calendarización de las fechas de entrega de los avances, columna III. En la mayoría de casos, las hipótesis observacionales en la IC se construyen a partir de los datos recogidos en campo. Son estas las que refuerzan, confirman o niegan nuestras conjeturas iniciales.

La columna II hace referencia a la relación secuencial y de concordancia entre los diferentes niveles de estudio. Cada aspecto debe dar soporte contextual y situacional al fenómeno, sujeto 0 actor social que se desea investigar. Si deseo investigar sobre el comercio informal en Gamarra, no solo me bastará con describir a los actores dentro de ese espacio; también se tendrá que describir cómo funciona el comercio en Gamarra y qué efectos tiene sobre otros actores del distrito.

La columna III indica que no existe un único momento o nivel específico para recoger información primaria. Que la respuesta a la pregunta general no solo se responde con trabajos previos, sino también con datos empíricos de campo. Sin embargo, para sustentar y dar respuestas al nivel específico el trabajo de campo sí es un requisito indispensable.

\section{Conclusiones}

La MCc es una propuesta de instrumento metodológico que sirve para estructurar y organizar el diseño de investigación con enfoque cualitativo. Es útil en la medida en que no parte de una lógica causalista lineal, ya que los fenómenos, sujetos o actores de estudio que pretende conocer, en última 
instancia, son el individuo y sus percepciones y acciones sobre los espacios donde interactúa, escapa a la rígida operativización de sus contenidos.

La MCc es una propuesta de instrumento metodológico que sirve para estructurar y organizar el diseño de investigación con enfoque cualitativo. Es útil en la medida en que no parte de una lógica causalista lineal que deviene del enfoque deductivo asociado a la matriz cuantitativa, sino que al estudiar los fenómenos sociocualitativos, dentro de los cuales están insertos los sujetos o actores de estudio que pretende conocer, parte del individuo, de sus percepciones y acciones sobre los espacios donde interactúa. Sirve para escapar a la rígida operativización de los contenidos cuantitativos y, por el contrario, dota de rigurosidad ética y fidelidad a las intenciones y significados de la vida de las comunidades y sus integrantes en procesos y problemáticas trascendentales para sus vidas.

La MCc es comprensiva y flexible de acuerdo con los tópicos que aborda la IC, ya que no obliga a depender exclusivamente con una hipótesis o marco teórico previo para emprender una investigación, pues durante el trabajo de campo emergen los primeros indicios para la construcción teórica de casos específicos o comparaciones plausibles de ser generalizables. En la MCc también se considera la inclusión de lo que se llaman niveles socioestructurales de estudio o dimensiones contextuales y situacionales, lo que permite tener un panorama de las dimensiones donde están inmersos los actores o sujetos que son parte de la investigación.

La metodología que se propone desde la MCc permite plantear dos formas de trabajo. En primer lugar, se sugiere escoger los métodos y las técnicas pertinentes para cada nivel donde se enmarca la investigación y el tipo de pregunta y objetivos que se desean alcanzar. En segundo lugar, postula que los datos e información obtenida durante el trabajo de campo no solo sirve para sustentar el nivel, la pregunta o el objetivo específico de la investigación, sino también para fortalecer y establecer los eslabones que conecta lo específico con y entre los niveles mesosocial y macrosocial. Esto es un punto diferencial en relación con la MCc.

\section{Contribuciones}

Mercedes P. Giesecke Sara Lafosse ha participado en la concepción, la recolección de datos, la redacción y la aprobación de la versión final del artículo.

\section{Fuentes de financiamiento}

Autofinanciado.

\section{Conflictos de intereses}

Ninguno. 


\section{REFERENCIAS BIBLIOGRÁFICAS}

Bernard, H. R. (1995). Métodos de investigación en antropología. Abordajes cualitativos y cuantitativos. Walnut Creek: Altamira Press.

Bourdieu, P., Chamboredon, J. C. y Passeron, J. C. (2002). El oficio del sociólogo. Presupuestos epistemológicos. Buenos Aires: Siglo XXI.

Carrasco, D. S. (2018). Metodología de la investigación científica. Pautas metodológicas para diseñar y elaborar el proyecto de investigación. Lima: San Marcos.

Coutinho, C. P. (2011). Metodología de investigação em ciências sociais e humanas: teoria e prática. Coimbra: Almedina.

Díaz, C., Flores, E. y Suárez, G. (2016). Guía de investigación en educación. Lima: Vicerrectorado de Investigación de la Pontificia Universidad Católica del Perú.

Giesecke, M. (2018a). Informe académico: Proyecto de investigación. Métodos cualitativos en estudios históricos sociales. Grupo de investigación: Diseñando el Perú: estado, ciudadanía, intelectuales y política. Universidad Nacional Mayor de San Marcos (mimeo).

Giesecke, M. (2018b). I Ciclo Internacional de Metodología Cualitativa para la Investigación en Ciencias Histórico Sociales: Enfoques, métodos y procedimientos. Facultad de Ciencias Sociales de la Universidad Mayor de San Marcos.

González Rey, F. (2008). Investigación cualitativa y subjetividad. Ciudad de Guatemala: Oficina de Derechos Humanos del Arzobispado de Guatemala.

Hernández, R., Fernández, C. y Batista, M. (2010). Metodología de investigación. Ciudad de México: McGraw-Hill e Interamericana.

Izcara, S. (2014). Manual de metodología cualitativa. Ciudad de México: Fontamara.

Marroquín, P. R. (2012). Matriz operacional de la variable y matriz de consistencia. Recuperado de http://www.une.edu.pe/diapositivas3-matrizde-consistencia-19-08-12.pdf

Murcia, N. y Jaramillo, L. (2003). Diseño metodológico de la investigación. En La complementariedad etnográfica. Investigación cualitativa. Una guía para abordar estudios sociales. Armenia: Kinesis.

Murcia, N. y Jaramillo, L. (2003). Una posibilidad de diseño metodológico desde la práctica reflexiva. En La complementariedad etnográfica. Investigación cualitativa. Una guía para abordar estudios sociales. Armenia: Kinesis.

Packer, M. (2013). La ciencia de la investigación cualitativa. Bogotá: Universidad de los Andes. 
Ruiz, J. I. (2012). Metodología de la investigación cualitativa. Bilbao: Universidad de Deusto.

Salgado, A. (2007). Investigación cualitativa: diseños, evaluación del rigor metodológico y retos. Liberabit, 13, pp. 71-78. Recuperado de http:// www.scielo.org.pe/scielo.php?script=sci_arttext\&pid=s172948272007000100009\&lng=es\&tlng=es

Sautu, R. (2005). Todo es teoría: objetivos y métodos de investigación. Buenos Aires: Lumiere.

Sierra, F. (1998). Función y sentido de la entrevista cualitativa en la investigación social. En J. Galindo Cáceres (Coord.), Técnicas de investigación en sociedad, cultura y comunicación (pp. 275-345). Ciudad de México: Pearson Educación.

Tamayo, M. (2003). El proceso de investigación científica. Ciudad de México: Limusa. 